\title{
MAPS OF CERTAIN CYCLIC INVOLUTIONS ON TWO-DIMENSIONAL CARRIERS
}

BY W. R. HUTCHERSON

1. Introduction. The following paper derives the fundamental properties of the involutions on an algebraic surface which have but a finite number of invariant points. Except for a few particular cases, they cannot be regarded as subcases of those having a curve of invariant points; they require one more equation for their definition, analogous to the singular correspondences on algebraic curves. They exist only on surfaces having particular moduli.

2. Discussion of $I_{n}$. Consider two surfaces $F(x)=0$ and $\Phi\left(x^{\prime}\right)=0$ with the property that any point $P$ on $F(x)=0$ uniquely fixes a point $P^{\prime}$ on $\Phi\left(x^{\prime}\right)=0$ and, conversely, the point $P^{\prime}$ fixes $n$ points $P_{1} \equiv P, P_{2}, \cdots, P_{n}$ on $F$. There is thus set up an $(n, 1)$ correspondence between the points of $F=0$ and $\Phi=0$. Now any one of the $n$ points $P_{1}, \cdots, P_{n}$ on $F=0$ definitely determines the whole group of $n$ points to which it belongs. Hence, it will be said that $F$ contains an involution $I_{n}$ of order $n$, and that this $I_{n}$ belongs to $\Phi\left(x^{\prime}\right)=0$.

There are two kinds of involutions; $F$ may contain one or more curves, each point of which contains two or more coincidences of these $n$ points $P_{1}, \cdots, P_{n}, P_{i}=P_{k}$. Such curves are called curves of coincidences. The surface $\Phi\left(x^{\prime}\right)=0$ then contains a locus of branch points in $(1,1)$ correspondence with the curve of coincidences on $F$. The other kind of involution is such that $F$ has only a finite number of coincident points. Thus, $\Phi\left(x^{\prime}\right)$ has in this case exactly the same number of branch points.

If $\Phi\left(x^{\prime}\right)=0$ is a rational surface, or a plane, $I_{n}$ is said to be rational. If $F(x)=0$ is rational, $\Phi\left(x^{\prime}\right)=0$ must be rational.* The converse is not true.

In this paper only $I_{n}$ on $F(x)=0$ with a finite number of coincident points will be considered. Such an $I_{n}$ can be gener-

* Castelnuovo, Mathematische Annalen, vol. 44 (1894), pp. 125-155. 
ated by a group of birational transformations of the surface $F(x)=0$ into itself. The involution is cyclic, abelian, etc. according as it is generated by a cyclic, abelian, etc. group of transformations. This group is, of course, cyclic if $n$ is prime. However, it may or may not be birational (Cremonian) for the whole space in which $F(x)=0$ lies.*

3. Space of $r$ Dimensions. In a space $S_{r}$ of $r$ dimensions, it is possible to reduce $F(x)=0$ and $\Phi\left(x^{\prime}\right)=0$ to their normal forms. A surface is said to be normal in a linear $S_{r}$ when it can not be obtained as the projection of a surface of the same order from a space $S_{m}, m>r$.

Call $T$ the cyclic transformation which generates $I_{n}$ (where $n$ is a prime) on $F(x)=0$, and $P$ a point of coincidence. Let $C$ be any curve on $F(x)=0$, through $P$. The image of $C$ under $T$ is another curve on $F$ through $P$. The point $P$ is called a perfect point of coincidence if $C$ and every image of $C$ touches the same line at $P$ for every tangent to $F$ at $P$. Otherwise $P$ is nonperfect. The corresponding branch point on $\Phi\left(x^{\prime}\right)=0$ is said to be perfect or non-perfect according as $P$ is a perfect or nonperfect point of coincidence.

When $F$ is reduced to its normal form, the operations of $I_{p}$ can be represented by a collineation of period $p$, under which $F$ is invariant in $S_{r}$. Since $p<r$, there exist not more than $p$ spaces of invariant points. The form of each transformation is $x_{i}=\theta^{s} x_{i}^{\prime}$, where $\theta^{p}=1$.

4. Discussion of $I_{2}$. Theorem 1. If $n=2$, every point $P$ of coincidence on $F(x)=0$ must be a perfect point.

Proof. It has been proved that, given an $I_{p}$, it can always be represented by a collineation in $S_{R}$, having $p$ axes, or spaces of invariant points, only one of which meets the surface $F$ which contains the involution.

When $p=2$ there are then only two axes. The transformation $T$ may be written

$$
\begin{aligned}
X_{1}^{\prime}: X_{2}^{\prime}: \cdots: X_{r+1}^{\prime}: X_{r+2}^{\prime}: \cdots: X_{r+8+2}^{\prime} \\
=X_{1}: X_{2}: \cdots: X_{r+1}: \epsilon X_{r+2}: \cdots: \epsilon X_{r+8+2},
\end{aligned}
$$

where $\epsilon=-1$.

*F. Enriques, Bologna Rendiconti, (2), vol. 14 (1910), pp. 71-75. 
Consider an invariant point $A$ on a surface $F$ in $S_{R}$, where $R=r+s+1$. Call the hyperplanes

$$
\sum_{i=1}^{r+1} s_{i} x_{i}^{\prime}=0, \Sigma^{(0)} \text {, and } \sum_{k=r+2}^{r+s+2} r_{k} x_{k}^{\prime}=0, \Sigma^{(1)} \text {. }
$$

Now $\Sigma^{(0)}$ contains one axis $S^{(1)}$ and $\Sigma^{(1)}$ the other $S^{(0)}$. Require $\Sigma^{(0)}$ to pass through point $A$ in axis $S^{(0)}$. It cuts $F$ in a system of invariant curves $|C|$, on each of which point $A$ is the only coincident point. The tangents to $|C|$ at point $A$ on $F$ all lie in one tangent plane. $\Sigma^{(0)}$ contains this plane. There are two invariant directions at $A$ in this plane or all are invariant. Call two invariant directions $a_{1}$ and $a_{2}$; then $a_{1}$ must have another invariant point $A_{1}$ on it. The point $A_{1}$ must lie in the other axis $S^{(1)}$ for $A$ is the only point of $S^{(0)}$ in the tangent plane. Likewise $a_{2}$ must have another invariant point $A_{2}$. It must also lie in $S^{(1)}$. A linear combination of the coordinates of $A_{1}$ and $A_{2}$ gives $\infty$ points on line $A_{1} A_{2}$, each of which is a coincident point (invariant point). Line $A_{1} A_{2}$ lies in axis $S^{(1)}$. Hence, by joining $A$ and every point on $A_{1} A_{2}$, we obtain $\infty$ invariant directions. Therefore point $A$ is a perfect point. This holds for a rational and an irrational surface.

Two illustrations of $I_{2}$ are given. The first one uses the quadratic transformation $x_{i}=1 / x_{i}^{\prime}$ in $S_{2}$ with four fixed points $( \pm 1, \pm 1,1)$. Nets* of cubic curves are mapped on the Cayley cubic surface with four nodes.

The second illustration maps a surface of Enriques of order six upon a double plane. The Cremona involution in $S_{3}$, $y_{i}=1 / y_{i}^{\prime}$, has eight fixed points $(1, \pm 1, \pm 1, \pm 1)$ on the surface $F$.

Several theorems follow, the proofs of which are omitted. $\dagger$

TheOREM 2. Consider on an algebraic surface $F$ a point $A$, which is non-perfect in a cyclic involution of third order under

* A. Emch, On the invariant net of cubics in the Steinerian transformation, this Bulletin, vol. 24 (1918), pp. 327-330. On plane algebraic curves which are invariant under a quadratic Cremona transformation, Tôhoku Mathematical Journal, vol. 21(1922), pp. 310-326.

$\dagger$ Earlier proofs of these theorems were also given by Godeaux. See Sisam, Involutions of irrational surfaces, Bulletin of the National Research Council, No. 63, vol. 14 (1928), pp. 295-309. Godeaux, Brussells Bulletin, 1927, pp. 524-543. 
which $F$ is invariant; it follows that in the domain of the first order of $A$, there are two distinct fixed points. These points are perfect coincidences of the involution.

THEOREM 3. Consider a cyclic involution of prime order, having only a finite number of fixed points, belonging to an algebraic surface $F$, and possessing a non-perfect fixed point adjacent to which are two perfect fixed points; then this involution is of order three.

A map of $I_{3}$ belonging to an irrational quintic surface upon an irrational surface in $S_{7}$, has been discussed. There are two perfect and five non-perfect coincidences.

5. Discussion of $I_{5}$ belonging to $F_{3}$ in $S_{3}$. Consider the surface $F_{3}\left(x_{1} x_{2} x_{3} x_{4}\right) \equiv a x_{1}^{2} x_{3}+b x_{2}^{2} x_{1}+c x_{3}^{2} x_{4}+d x_{4}^{2} x_{2}=0$, in $S_{3}$, invariant under the cyclic collineation $T$ of order five

$$
x_{1}^{\prime}: x_{2}^{\prime}: x_{3}^{\prime}: x_{4}^{\prime}=x_{1}: \epsilon^{1} x_{2}: \epsilon^{2} x_{3}: \epsilon^{3} x_{4}, \quad\left(\epsilon^{5}=1\right) .
$$

There are four invariant axes $S^{(0)}, S^{(1)}, S^{(2)}, S^{(3)}$ each consisting of a point: $P_{1} \equiv(1,0,0,0), P_{2} \equiv(0,1,0,0), P_{3} \equiv(0,0,1,0)$, and $P_{4} \equiv(0,0,0,1)$. Each lies on the surface $F$, and since these are the only possible invariant axes, the surface $F$ has only four points of coincidence.

Consider a curve $C$, not transformed into itself by $T$, and passing through $P_{1}$. Take a plane $x_{3}+\lambda x_{4}=0$ of the pencil passing through $P_{1}$ and $P_{2}$, tangent to $C$. This plane $x_{3}+\epsilon \lambda x_{4}$ $=0$ by $T$ and hence is non-invariant. The curve cut out on $F$ by $x_{3}+\lambda x_{4}=0$ is therefore non-invariant. The common tangent to the two curves is not transformed into itself. Hence, the two curves do not touch each other at $P_{1}$. Since $C$ was a variable curve through $P_{1}$ satisfying the non-invariant property, it follows that $P_{1}$ is a non-perfect coincidence point. A similar argument shows that $P_{2}, P_{3}$, and $P_{4}$ are also non-perfect coincidence points. The following theorem is proved.

THEOREM 4. The $I_{5}$ belonging to $F_{3}$ in $S_{3}$ has four non-perfect points of coincidence.

Consider the complete system cut out on $F$ by the quintic surfaces. Let $|A|$ be the system. Its dimension is 55 , its genus is 31 , and the number of variable intersections of two members of the system is 75 . A curve $A$ of this system is not in general transformed into itself by $T$. There are, however, five partial systems 
in $|A|$ which are transformed into themselves. Call these $\left|A_{1}\right|$, $\left|A_{2}\right|,\left|A_{3}\right|,\left|A_{4}\right|$, and $\left|A_{5}\right|$. By use of $\left|A_{1}\right|$, we find

$$
\begin{aligned}
a_{11111} x_{1}^{5} & +a_{22222} x_{2}{ }^{5}+a_{33333} x_{3}^{5}+a_{44444} x_{4}{ }^{5}+a_{12444} x_{1} x_{2} x_{4}{ }^{3} \\
& +a_{12223} x_{1} x_{2}^{3} x_{3}+a_{13344} x_{1} x_{3}^{2} x_{4}^{2}+a_{11233} x_{1}{ }^{2} x_{2} x_{3}{ }^{2} \\
& +a_{11224} x_{1}{ }^{2} x_{2}^{2} x_{4}+a_{11134} x_{1}^{3} x_{3} x_{4}+a_{23334} x_{2} x_{3}{ }^{3} x_{4} \\
& +a_{22344} x_{2}{ }^{2} x_{3} x_{4}{ }^{2}=0 .
\end{aligned}
$$

We refer the curves $A_{1}$ projectively to the hyperplanes of a linear space of eleven dimensions. We obtain a surface $\Phi$, of order 15, with hyperplane sections of genus 7 , as the image of $I_{5}$.

The equations of the transformation for mapping $I_{5}$ upon $\Phi$ in $S_{11}$ are

$$
\begin{array}{llll}
\rho X_{1}=x_{1}^{5}, & \rho X_{4}=x_{4}{ }^{5}, & \rho X_{7}=x_{1} x_{3}^{2} x_{4}^{2}, & \rho X_{10}=x_{1}^{3} x_{3} x_{4}, \\
\rho X_{2}=x_{2}{ }^{5}, & \rho X_{5}=x_{1} x_{2} x_{4}^{3}, & \rho X_{8}=x_{1}^{2} x_{2} x_{3}{ }^{2}, & \rho X_{11}=x_{2} x_{3}^{3} x_{4}, \\
\rho X_{3}=x_{3}{ }^{5}, & \rho X_{6}=x_{1} x_{2}{ }^{3} x_{3}, & \rho X_{9}=x_{1}{ }^{2} x_{2}{ }^{2} x_{4}, & \rho X_{12}=x_{2}^{2} x_{3} x_{4}^{2} .
\end{array}
$$

By eliminating $\rho, x_{1}, x_{2}, x_{3}, x_{4}$ from these twelve equations and from $F_{3}\left(x_{1} x_{2} x_{3} x_{4}\right)=0$, we get as the nine equations defining the surface $\Phi$,

$$
\begin{aligned}
\left\|\begin{array}{ccc}
X_{4} & X_{5} & X_{12} \\
X_{5} & X_{9} & X_{6}
\end{array}\right\|=0, & \left\|\begin{array}{lll}
X_{2} & X_{9} & X_{12} \\
X_{6} & X_{10} & X_{7}
\end{array}\right\|=0,\left\|\begin{array}{ll}
X_{1} X_{10} X_{8} \\
X_{10} X_{7} & X_{11}
\end{array}\right\|=0, \\
& \left\|\begin{array}{lll}
X_{3} & X_{11} & X_{7} \\
X_{11} & X_{12} & X_{5}
\end{array}\right\|=0,
\end{aligned}
$$

and $a X_{8}+b X_{6}+c X_{11}+d X_{12}=0$. Designate by $P_{1}^{\prime}$ the branch point of $\Phi$ corresponding to the point $P_{1}$ on $F$. The coordinates of $P_{1}^{\prime}$ are all zero except $X_{1}$.

The curves $A_{1}$ on $F$ pass through $P_{1}$ if $a_{11111}=0$. The tangent plane at $P_{1}$ to $F$ is $x_{3}=0$. Now, the system of quintic surfaces passing through $P_{1}$ cuts $x_{3}=0$ in the curves $x_{3}=0, a_{22222} x_{2}{ }^{5}$ $+a_{44444} x_{4}^{5}+a_{12444} x_{1} x_{2} x_{4}^{8}+a_{11224} x_{1}{ }^{2} x_{2}{ }^{2} x_{4}=0$. For general values of the constants this is a quintic curve with a triple point at $P_{1}$, two branches being tangent to the line $x_{2}=x_{3}=0$ and one to the line $x_{3}=x_{4}=0$. When $a_{12444}=a_{11224}=0$, the plane quintic curve breaks up into five lines through $P_{1}$. These are all distinct except when either $a_{22222}=0$ or $a_{44444}=0$, when they coincide with $x_{3}=x_{4}=0$ or $x_{2}=x_{3}=0$, respectively. Since $P_{1}$ is non- 
perfect, the $\left|A_{1}\right|$ through $P_{1}$ must have five distinct branches, unless each branch touches one of the two invariant directions.

In the plane $x_{3}=0$ the involution $I_{5}$ is generated by the homography $T_{1}$, which is $x_{1}^{\prime}: x_{2}^{\prime}: x_{4}^{\prime}=x_{1}: \epsilon x_{2}: \epsilon^{3} x_{4}$. By use of the plane quadratic transformation $S$, which is $x_{1}: x_{2}: x_{4}=z_{1}^{2}: z_{1} z_{2}: z_{2} z_{4}$ and its inverse $z_{1}: z_{2}: z_{4}=x_{1} x_{2}: x_{2}^{2}: x_{1} x_{4}$, we can investigate the character of the adjacent invariant points along the two invariant directions at $P_{1}$. By the application of $S T_{1} S^{-1} \equiv T_{1}^{\prime}$,

or

$$
\left(z_{1}, z_{2}, z_{4}\right)^{S_{-1}} \sim\left(x_{1} x_{2}, x_{2}, x_{1} x_{4}\right)^{T_{1}} \sim\left(\epsilon x_{1} x_{2}, \epsilon^{2} x_{2}^{2}, \epsilon^{3} x_{1} x_{4}\right),
$$

$$
\left(x_{1} x_{2}, \epsilon x_{2}^{2}, \epsilon^{2} x_{1} x_{4}\right)^{S} \sim\left(z_{1}, \epsilon z_{2}, \epsilon^{2} z_{4}\right) .
$$

Thus the new transformation $T_{1}^{\prime}$ is $x_{1}^{\prime}: x_{2}^{\prime}: x_{4}^{\prime}=x_{1}: \epsilon x_{2}: \epsilon^{2} x_{4}$. The invariant point adjacent to $P_{1}$ along the line $x_{3}=x_{4}=0$ is still a non-perfect coincidence point. Investigate the next point by use of $S T_{1}^{\prime} S^{-1} \equiv T_{1}^{\prime \prime}\left(z_{1}, z_{2}, z_{4}\right) \sim\left(z_{1}, \epsilon z_{2}, \epsilon z_{4}\right)$. This point is a perfect point of coincidence.

By use of another quadratic transformation $R$, namely $x_{1}: x_{2}: x_{4}=z_{1}^{2}: z_{2} z_{4}: z_{1} z_{4}$ and its inverse $z_{1}: z_{2}: z_{4}=x_{1} x_{4}: x_{1} x_{2}: x_{4}^{2}$, the adjacent point to $P_{1}$ along $x_{2}=x_{3}=0$ can be investigated. Applying $R T_{1} R^{-1}$ as above, we have $\left(z_{1}, z_{2}, z_{4}\right) \sim\left(\epsilon^{2} z_{1}, z_{2}, z_{4}\right)$. Hence we get a perfect coincidence point. The following theorem is proved.

THEOREM 5. The non-perfect coincidence point $P_{1}$ on $F$ has one adjacent perfect point along the line $x_{2}=x_{3}=0$, a non-perfect one along the line $x_{3}=x_{4}=0$, with a perfect one adjacent to this.

The tangent plane to $F$ at $P_{2}(0,1,0,0)$ is $x_{1}=0$. The homography $T_{2}$ in $x_{1}=0$ is $x_{2}^{\prime}: x_{3}^{\prime}: x_{4}^{\prime}=x_{2}: \epsilon x_{3}: \epsilon^{2} x_{4}$. Apply $S T_{2} S^{-1}$ and proceed as above. We find $x_{2}^{\prime}: x_{3}^{\prime}: x_{4}^{\prime}=x_{2}: \epsilon x_{3}: \epsilon x_{4}$. Hence, the adjacent point along $x_{1}=x_{3}=0$ is perfect. By use of $R T_{2} R^{-1}$ we obtain $\left(z_{2}, z_{3}, z_{4}\right) \sim\left(z_{2}, z_{3}, \epsilon_{3} z_{4}\right)$. This indicates a non-perfect point. By use of $R T_{2}^{\prime} R^{-1}$, we get $x_{2}^{\prime}: x_{3}^{\prime}: x_{4}^{\prime}=\epsilon^{3} x_{2}: x_{3}: x_{4}$. This gives a perfect point. Hence we may state the following theorem.

THEOREM 6. The non-perfect coincidence point $P_{2}$ on $F$ has one adjacent point along the line $x_{1}=x_{4}=0$, a non-perfect adjacent one along $x_{1}=x_{3}=0$, with a perfect one adjacent to this.

The point $P_{3}(0,0,1,0)$ has $x_{4}=0$ for its tangent plane. The homography becomes, in this tangent plane, $T_{3}: x_{1}^{\prime}: x_{2}^{\prime}: x_{3}^{\prime}$ 
$=x_{1}: \epsilon x_{2}: \epsilon^{2} x_{3}$. Introduce two quadratic transformations for use in discovering the nature of $P_{3}(0,0,1,0)$. Calling the first one $U$ and the second $V$, we have

$$
\begin{aligned}
U: & y_{1}: y_{2}: y_{3}=w_{2} w_{3}: w_{1} w_{2}: w_{3}^{2}, \\
U^{-1}: & w_{1}: w_{2}: w_{3}=y_{2} y_{3}: y_{1}^{2}: y_{1} y_{3}, \\
V: & y_{1}: y_{2}: y_{3}=w_{1} w_{2}: w_{1} w_{3}: w_{2}^{2}, \\
V^{-1}: & w_{1}: w_{2}: w_{3}=y_{1}^{2}: y_{1} y_{3}: y_{2} y_{3} .
\end{aligned}
$$

The adjacent points along the line $x_{4}=x_{2}=0$ compel the use of $U T_{3} U^{-1} \equiv T_{3}^{\prime}$, then $V T_{3}^{\prime} V^{-1}$ or $T_{3}^{\prime \prime}$, and then $U T_{3}^{\prime \prime} U^{-1}$ before a perfect point is found. We have $\left(w_{1}, w_{2}, w_{3}\right) \sim\left(\epsilon_{3} w_{1}, w_{2}, \epsilon^{2} w_{3}\right)$. This point is non-perfect. Consider $\left(w_{1}, w_{2}, w_{3}\right) \sim\left(\epsilon^{3} w_{1}, w_{2}, \epsilon^{2} w_{3}\right)$. This also is non-perfect.

Consider $\left(w_{1}, w_{2}, w_{3}\right) \sim\left(\epsilon^{2} w_{1}, \epsilon^{2} w_{2}, \epsilon^{2} w_{3}\right)$. This is a perfect point in the neighborhood of $P_{3}$ of the third order.

Now consider the possibilities along $x_{4}=x_{1}=0$, an invariant direction. Consider $\left(w_{1}, w_{2}, w_{3}\right) \sim\left(w_{1}, \epsilon^{2} w_{2}, \epsilon^{3} w_{3}\right)$. This is a nonperfect point. We have $\left(w_{1}, w_{2}, w_{3}\right) \sim\left(\epsilon^{2} w_{1}, \epsilon^{2} w_{2}, \epsilon^{3} w_{3}\right)$. Hence we may state the following theorem.

THEOREM 7. The non-perfect coincidence point $P_{3}$ on $F$ has no adjacent perfect point of coincidence. There is one perfect point in the domain of the second order of $P_{3}$ and another in the domain of the third order of $P_{3}$.

The point $P_{4}(0,0,0,1)$ has the plane $x_{2}=0$ for its tangent plane. The homography $T$ in this plane becomes $T_{4}: x_{1}^{\prime}: x_{3}^{\prime}: x_{4}^{\prime}$ $=x_{1}: \epsilon^{2} x_{3}: \epsilon^{3} x_{4}$. Consider the direction $x_{3}=x_{2}=0$ at $P_{4}$. We have $\left(w_{1}, w_{3}, w_{4}\right) \sim\left(w_{1}, w_{3}, \epsilon^{3} w_{4}\right)$. Hence, the adjacent point is perfect along $x_{2}=x_{3}=0$. Now $\left(w_{1}, w_{3}, w_{4}\right) \sim\left(w_{1}, \epsilon^{3} w_{3}, w_{4}\right)$ and $\left(w_{1}, w_{3}, w_{4}\right)$ $\sim\left(w_{1}, w_{3}, \epsilon^{3} w_{4}\right)$. Hence we have the following theorems.

THEOREM 8. The non-perfect coincidence point $P_{4}$ has an adjacent perfect point along the line $x_{2}=x_{3}=0$, a non-perfect one along the line $x_{1}=x_{2}=0$, with a perfect one adjacent to this.

TheOREM 9. The system of invariant curves cut upon $F$ by surfaces of degree lower than five all pass through the four coincidence points along the invariant directions. The number of branches through each point is less than five.

Cornell University 